\title{
Rhythm Disorders in Endocrinology
}

\author{
Gagan Priya, ${ }^{1}$ Shibba Takkar Chhabra ${ }^{2}$ and Sanjay Kalra ${ }^{3}$ \\ 1. Department of Endocrinology, Fortis Hospital and Ivy Hospital, Mohali, Punjab, India; 2. Department of Cardiology, Dayanand Medical \\ College and Hospital, Ludhiana, Punjab, India; 3. Department of Endocrinology, Bharti Hospital, Karnal, Haryana, India
}

DOI: https://doi.org/10.17925/EJAE.2021.7.1.12

\begin{abstract}
$\mathrm{C}$ ardiac rhythm is closely regulated by the neuroendocrine axis, with an intricate balance between the sympathetic and parasympathetic nervous systems. In addition, various cardiac reflexes and hormones mediate changes in heart rate, cardiac conduction and contractility to meet the oxygen and nutritional demands of the body. Endocrine and metabolic syndromes can cause rhythm disorders, both as a direct result of hormone excess or deficiency states, or indirectly due to structural or ischaemic heart disease and electrolyte or mineral imbalance. This calls for a need for endocrine and metabolic vigilance in patients presenting with rhythm or conduction abnormalities, as the identification and appropriate management of underlying endocrinopathy can significantly reduce the risk of arrhythmias. At the same time, cardiac vigilance is required during the management of endocrine disorders, such as diabetes mellitus, acromegaly, thyrotoxicosis, pheochromocytoma, Cushing's syndrome, primary hyperaldosteronism and hyperparathyroidism. While these disorders are associated with an increased risk of ischaemic heart disease, heart failure and cardiomyopathy, the risk of arrhythmias is also increased. In addition, endocrine therapies may impact the arrhythmic risk. Therefore, there is a need for close collaboration between the endocrinologist and the cardiologist.
\end{abstract}

\section{Keywords}

Cardiac arrhythmias, diabetes, thyrotoxicosis, amiodarone-induced thyroid dysfunction, pheochromocytoma, acromegaly

Disclosures: Gagan Priya, Shibba Takkar Chhabra and Sanjay Kalra have no financial or non-financial relationships or activities to declare in relation to this article.

Review process: Double-blind peer review.

Compliance with ethics: This study involves a review of the literature and did not involve any studies with human or animal subjects performed by any of the authors.

Authorship: The named authors meet the International Committee of Medical Journal Editors (ICMJE) criteria for authorship of this manuscript, take responsibility for the integrity of the work as a whole, and have given final approval for the version to be published.

Access: This article is freely accessible at

touchCARDIO.com (C) Touch Medical Media 2021

Received: 10 November 2020

Accepted: 8 April 2021

Published online: 7 June 2021

Citation: European Journal of Arrhythmia

\& Electrophysiology. 2021;7(1):12-8

Corresponding author: Gagan Priya, Department of

Endocrinology, Fortis Hospital, Sector 62, SAS Nagar,

Mohali - 160062, Punjab, India

E: gpriya77@gmail.com

Support: No funding has been received

for the publication of this article.
Several closely regulated ion channels are involved in the various phases of the myocardial action potential (phases 0-4) (Figure 1). ${ }^{1}$ In the absence of neuroendocrine and other regulatory signals, the pacing rate of the sinoatrial node is 100 beats per minute (bpm). However, heart rate is not fixed, and varies along with cardiac output in order to meet the oxygen and nutritional demands of the body. ${ }^{2}$ This is brought about by cardiac reflexes (proprioceptor reflex, baroreceptor reflex and chemoreceptor reflex) and neuroendocrine regulation.

Any abnormality in the neuroendocrine system or $\mathrm{pH}$, partial pressure of oxygen or electrolyte balance, or in the auto-rhythmicity and conductivity of the cardiac conduction system can result in rhythm abnormalities. ${ }^{3-4}$ In this article, we focus on the neuroendocrine regulation of cardiac rhythm and rhythm disorders in various endocrine disorders.

\section{Neuroendocrine regulation of cardiac rhythm}

Two paired cardio-regulatory centres in the medulla oblongata receive inputs from the various parts of the body and send signals to regulate cardiac activity. ${ }^{3}$ The first of these is the cardioaccelerator region, which stimulates heart activity via the sympathetic nervous system. Norepinephrine is the dominant neurotransmitter at the sympathetic nervous system nerve endings. It opens the ligand-gated $\mathrm{Na}^{+}$and $\mathrm{Ca}^{2+}$ channels, allowing an influx of positively charged ions; thereby, shortening repolarization and increasing the rate of depolarization. ${ }^{3}$ Thus, norepinephrine has a positive chronotropic (increases heart rate), inotropic (increases contractility) and dromotropic (increases speed of conduction) effect. The second cardio-regulatory centre is the cardioinhibitory region, which sends inputs to the heart via the parasympathetic nervous system. Acetylcholine is the predominant neurotransmitter and it has effects opposite to that of norepinephrine. Acetylcholine opens ligand-gated $\mathrm{K}^{+}$channels, slows spontaneous depolarization and extends the repolarization phase. ${ }^{3}$ During rest, both the sympathetic and parasympathetic systems provide slight stimulation with predominance of vagal stimulation. Therefore, the heart beats at a usual rate of 72-75 bpm (below the sinoatrial node pacing rate of $100 \mathrm{bpm}$ ). ${ }^{3}$

Several factors may increase or decrease the heart rate and myocardial contractility (Table 1). Tachycardia may occur as a physiological response (in newborns, or during pregnancy, exercise or stress), or during fever, anaemia, hypoxia or endocrine disorders (thyrotoxicosis, catecholamine-producing tumours, cardiomyopathy and heart failure). On the other hand, the causes of bradycardia include defects in sinoatrial or atrioventricular node, ischaemia, endocrine and metabolic disorders (hyperkalaemia, hypothyroidism), and medications such as beta blockers or autonomic neuropathy. ${ }^{2}$ 
Figure 1: Phases of myocardial action potential

\begin{tabular}{|l|ll|}
\hline & \\
\hline & & \\
\hline
\end{tabular}

Cav1.2 = calcium channel, voltage-dependent, $L$ type, alpha $1 \mathrm{C}$ subunit; $h E R G=$ human ether-à-go-go-related gene; ICa = voltage gated calcium current; $I K 1=$ inward rectifier $K+$ current; $I K r=$ rapidly activating $K+$ current; $I K S$ = slowly activating $K+$ current; Ina = sodium current; Ito = transient outward $K V$ current; $K C N E 1=$ potassium voltage-gated channel subfamily E member 1: KCNE2 = potassium voltage-gated channel subfamily $E$ member 2; KCNQ1 = potassium voltage-gated channel subfamily Q member 1; Kir2.X = inward rectifier potassium channel; KV4.3 = potassium voltage-gated channel subfamily D member 3; UK = potassium current.

\section{Rhythm disturbances in endocrine disorders}

Normal endocrine and metabolic function are essential for cardiovascular health. While the relationship between ischaemic cardiovascular disease (CVD) or heart failure and endocrine disorders has been well documented in patients with diabetes mellitus, acromegaly, thyrotoxicosis, pheochromocytoma and hyperaldosteronism, ${ }^{2}$ there is less focus on rhythm disturbances in these patients. Rhythm disturbances can occur due to a variety of reasons in endocrinopathies: ${ }^{5}$

- hypofunction or hyperfunction of particular endocrine axes (thyroid or adrenal medulla);

- abnormalities in mineral and electrolyte balance (potassium, calcium, magnesium or sodium);

- secondary to atherosclerotic CVD;

- structural heart disease, such as cardiomyopathy or heart failure;

- drug-induced (antidiabetic agents that cause QT prolongation, such as teneligliptin); and

- coexistent inherited syndromes $\left(\mathrm{K}^{+}\right.$channelopathies in hyperthyroidism).

\section{Diabetes mellitus}

The risk of arrhythmias is increased in people with diabetes due to multiple factors, including concomitant hypertension, CVD, obesity, obstructive sleep apnoea and heart failure (Figure 2). In addition, both hyperglycaemia and hypoglycaemia significantly increase the risk of rhythm disturbances. ${ }^{1}$ Diabetes may also be associated with cardiac autonomic neuropathy and reduced heart rate variability. ${ }^{4}$

Many studies report an increased risk of atrial fibrillation in patients with diabetes. ${ }^{2}$ Atrial fibrillation is more common in those with longer duration of diabetes and poor glycaemic control (higher glycated haemoglobin [HbA1C]). ${ }^{6}$ In the ORBIT-AF registry, almost $30 \%$ of patients with atrial fibrillation had underlying diabetes, and these individuals were younger and more likely to have associated comorbidities (hypertension, heart
Table 1: Factors that increase or decrease the heart rate and myocardial contractility

\begin{tabular}{|c|c|c|}
\hline Factor & Increases heart rate & Decreases heart rate \\
\hline $\begin{array}{l}\text { Cardio-regulatory } \\
\text { region }\end{array}$ & $\begin{array}{l}\text { Release of norepinephrine } \\
\text { from sympathetic nerve } \\
\text { endings }\end{array}$ & $\begin{array}{l}\text { Release of acetylcholine } \\
\text { via parasympathetic nerve } \\
\text { endings }\end{array}$ \\
\hline Proprioceptors & $\begin{array}{l}\text { Increased rate during } \\
\text { exercise }\end{array}$ & $\begin{array}{l}\text { During recovery from } \\
\text { exercise }\end{array}$ \\
\hline Chemoreceptors & $\begin{array}{l}\text { Decreased oxygen } \\
\text { saturation, } \\
\text { Increased hydrogen ions, } \\
\mathrm{CO}_{2} \text { or lactic acid }\end{array}$ & $\begin{array}{l}\text { Decreased hydrogen and } \\
\mathrm{CO}_{2}\end{array}$ \\
\hline Baroreceptors & $\begin{array}{l}\text { Falling blood pressure or } \\
\text { volume }\end{array}$ & $\begin{array}{l}\text { Raised blood pressure or } \\
\text { volume }\end{array}$ \\
\hline Limbic system & $\begin{array}{l}\text { Emotions such as fear or } \\
\text { anxiety }\end{array}$ & Relaxation \\
\hline Catecholamines & $\begin{array}{l}\text { Increased secretion } \\
\text { of norepinephrine/ } \\
\text { epinephrine }\end{array}$ & Decreased secretion \\
\hline Thyroid hormones & Thyrotoxicosis & Hypothyroidism \\
\hline Sodium & Hyponatraemia & Hypernatraemia \\
\hline Potassium & Hypokalaemia & Hyperkalaemia \\
\hline Calcium & Hypercalcaemia & Hypocalcaemia \\
\hline Body temperature & Fever & Hypothermia \\
\hline
\end{tabular}

failure, chronic kidney disease, CVD or stroke).? Atrial fibrillation is a marker of poorer prognosis in individuals with diabetes, and is associated with greater morbidity and mortality. ${ }^{5-6}$ Therefore, the occurrence of atrial fibrillation in diabetes calls for detailed evaluation and prompt, aggressive, multifaceted risk-factor management.

Diabetes is also associated with reduced heart rate variability. ${ }^{8}$ In addition, several studies suggest that the QT interval is prolonged in both type 1 and type 2 diabetes. ${ }^{8}$ QT prolongation may occur during hypoglycaemia or acute coronary syndrome, or as a result of certain medications (teneligliptin or hydroxychloroquine).9-10 This predisposes individuals with diabetes to ventricular arrhythmias. Sudden cardiac death appears to be more common in people with diabetes compared with non-diabetic individuals. ${ }^{8}$ Ventricular arrhythmias have been associated with both hyperglycaemia and hypoglycaemia. ${ }^{2}$ In studies using continuous glucose monitoring and 24-hour Holter monitoring, hypoglycaemia, especially nocturnal hypoglycaemia, was associated with greater risk of bradycardia, atrial ectopy and ventricular arrhythmias. ${ }^{5}$ In addition, glycaemic variability may cause significant oxidative stress, another factor that predisposes to arrhythmogenesis.

Among anti-diabetic medications, there is a risk of QT prolongation related to the use of drugs if they result in hypoglycaemia (such as sulfonylureas or insulin), or as a direct effect of the drug (teneligliptin, hydroxychloroquine). Glucagon-like polypeptide-1 (GLP-1) receptor agonists cause an increase in heart rate (2-4 bpm). ${ }^{11}$ This effect seems to be due to increased sympathetic activity or direct activation of atrial GLP-1 receptors, but was not associated with any adverse cardiovascular effects or arrhythmias in long-term cardiovascular outcome trials. ${ }^{12}$ However, a recent meta-analysis suggested an increased risk of atrial fibrillation with the use of albiglutide, but not with the other GLP-1 receptor agonists. ${ }^{12}$ Therefore, all those with diabetes who have symptoms such as palpitations, light-headedness, presyncope or syncope 


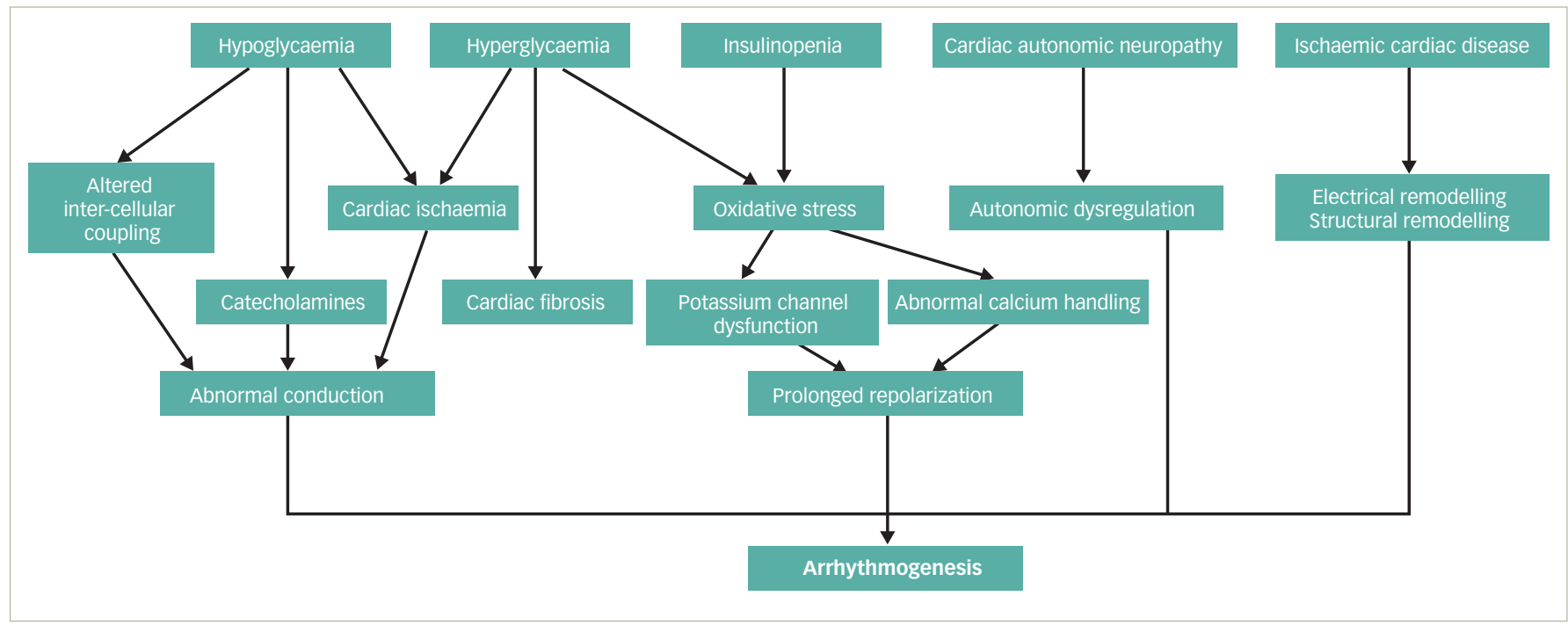

should undergo screening and diagnostic evaluation for arrhythmias. A baseline electrocardiogram (ECG) may be followed by 24-hour Holter monitoring if there is high suspicion. ${ }^{5}$

Prevention of arrhythmias requires multifaceted risk-factor management. Glycaemic targets should be individualized based on underlying risk of hypoglycaemia and comorbidities. More intensive glycaemic control increases the risk of hypoglycaemia, and therefore, less stringent targets for glycaemic control (HbA1C 7-8\%) should be considered in those with long-standing diabetes, history of recurrent or severe hypoglycaemia, advanced micro- or macro-vascular complications, comorbid conditions such as chronic kidney or liver disease, or limited life expectancy. Appropriate management of $\mathrm{CV}$ risk factors and weight is also important.

\section{Thyroid disorders}

Thyroid hormones regulate cardiac rhythm both directly and indirectly. T3 has genomic, as well as non-genomic, effects on the cardiovascular system. It regulates genes related to pacemaker activity and cardiac contractility, such as sarco/endoplasmic reticulum $\mathrm{Ca}^{2+}$ ATPase (SERCA2), phospholamban and myosin heavy chain kinase. ${ }^{2} \mathrm{~T} 3$ also regulates several myocardial ion transporters, including $\mathrm{Na}^{+}-\mathrm{K}^{+}$ATPase, $\mathrm{Na}^{+}-\mathrm{Ca} 2^{+}$exchanger, KV1.5 and KV4.2.2. In addition, T3 augments the effects of epinephrine and norepinephrine via upregulation of $\beta 1$ adrenergic receptors. ${ }^{2}$ In net, $T 3$ has positive inotropic, chronotropic and dromotropic effects.

\section{Thyrotoxicosis}

Thyroid hormone excess increases the risk of tachyarrhythmias via several mechanisms (Table 2). In addition to sinus tachycardia due to chronotropic and dromotropic effect of excess T3, there is also increased atrial ectopic activity and greater likelihood of re-entry as the effective refractory period shortens. Increased left ventricular mass and impaired ventricular relaxation also increases left atrial pressure. ${ }^{2}$ An ECG may demonstrate sinus tachycardia, increased voltage of the QRS complex, atrial and ventricular premature beats, and left ventricular hypertrophy and strain pattern. ${ }^{13}$ Paroxysmal atrial tachycardia and atrial fibrillation occur in approximately 6-12\% patients with hyperthyroidism. ${ }^{2}$

Thyrotoxicosis is the most common treatable cause of atrial fibrillation, and all patients with atrial fibrillation should be evaluated for thyroid dysfunction. ${ }^{5}$ Atrial fibrillation may be paroxysmal or persistent.
Table 2: Mechanisms of increased risk of tachyarrhythmias in thyrotoxicosis

\begin{tabular}{|l|l|}
\hline Mechanism & Effect in thyrotoxicosis \\
\hline Chronotropic effect & Increased heart rate \\
\hline Dromotropic effect & Increased rate of conduction \\
\hline Re-entry & Shortened effective refractory period \\
\hline Atrial ectopy & Increased \\
\hline $\begin{array}{l}\text { Increased left atrial } \\
\text { pressure }\end{array}$ & $\begin{array}{l}\text { Increased left ventricular mass and impaired } \\
\text { relaxation }\end{array}$ \\
\hline Cardiac ischaemia & Due to increased heart rate \\
\hline
\end{tabular}

Patients who are at increased risk of atrial fibrillation include the elderly, men, and those with hypertension, congestive heart failure, ischaemic heart disease or valvular heart disease (such as mitral valve prolapse)..$^{14}$

The correction of the thyrotoxic state can reverse atrial fibrillation in nearly $55-75 \%$ patients, though this may take 4-6 months. ${ }^{5}$ Therefore, while beta blockers are used for rate control and symptom management, the management of thyrotoxicosis and the restoration of the euthyroid state is very important.13 Patients who are older, have structural or ischaemic heart disease or have a long duration of atrial fibrillation are more likely to develop persistent atrial fibrillation. In such cases, cardioversion should be considered if the atrial fibrillation persists after 6 months of achieving a stable euthyroid state.

While overt hyperthyroidism is clearly associated with increased risk of atrial fibrillation, patients with subclinical hyperthyroidism are also at threefold increased risk of atrial fibrillation. This is especially true for elderly patients and women who are postmenopausal. ${ }^{5}$ Therefore, subclinical hyperthyroidism should be evaluated and treated in patients who have serum thyroid-stimulating hormone levels $<0.1 \mathrm{mIU} / \mathrm{L}$, are elderly, postmenopausal women or have CVD or osteoporosis. In addition, all individuals presenting with tachyarrhythmias should undergo thyroid evaluation to exclude overt or subclinical thyrotoxicosis even if they do not have other signs or symptoms of thyrotoxicosis.

The risk of stroke is increased in patients with hyperthyroidism with persistent atrial fibrillation, as hyperthyroidism is associated with 
Table 3: Amiodarone-induced thyrotoxicosis

\begin{tabular}{|l|l|l|}
\hline & Amiodarone-induced thyrotoxicosis type 1 & Amiodarone-induced thyrotoxicosis type 2 \\
\hline Pathogenesis & $\begin{array}{l}\text { Iodine-induced hyperthyroidism } \\
\text { Underlying nodular thyroid disease or latent Graves' disease }\end{array}$ & $\begin{array}{l}\text { Destructive thyroiditis } \\
\text { Previously apparently normal thyroid }\end{array}$ \\
\hline Radioiodine uptake & $\begin{array}{l}\text { Low or normal radioactive iodine uptake test (usually not very } \\
\text { high, unlike in Graves' disease, due to iodine load) }\end{array}$ & Suppressed uptake \\
\hline Ultrasonography with colour Doppler & Increased vascularity & Absent hypervascularity \\
\hline (99m)Tc-sestaMIBI scan & Increased uptake & Decreased uptake \\
\hline Treatment & $\begin{array}{l}\text { Anti-thyroid drugs } \\
\text { Beta blockers } \\
\text { Potassium perchlorate } \\
\text { Thyroidectomy (rare) }\end{array}$ & $\begin{array}{l}\text { Glucocorticoids } \\
\text { Beta blockers } \\
\text { Thyroidectomy (rare) }\end{array}$ \\
\hline
\end{tabular}

coagulation abnormalities. ${ }^{2}$ However, the risk of stroke is lower compared with non-hyperthyroid subjects with persistent atrial fibrillation. ${ }^{5}$ The decision to treat with long-term oral anticoagulants should be made based on the risk of stroke as assessed by the $\mathrm{CHA}_{2} \mathrm{DS}_{2}$-VASC score. Ventricular arrhythmias, on the other hand, are rare in patients with hyperthyroidism. However, certain channelopathies associated with Graves' disease may result in long QT syndrome and risk for ventricular arrhythmias. ${ }^{1}$

\section{Hypothyroidism}

Hypothyroidism is associated with slowing of the heart rate and conduction of electric stimuli along the cardiac conduction pathway. ${ }^{2}$ On an ECG, patients with overt hypothyroidism may manifest sinus bradycardia, low voltage QRS complex, prolongation of PR and QT intervals and flattened or inverted T waves. ${ }^{15}$ Varying degrees of conduction blocks may be seen, and 20-30\% of cases of severe untreated hypothyroidism may have QT prolongation. However, torsades de pointes, ventricular tachycardia and ventricular fibrillation are rare. ${ }^{15}$ Most rhythm and conduction abnormalities seen in hypothyroidism resolve promptly with initiation of levothyroxine therapy in appropriate doses.

Hypothyroidism is also associated with increased CV risk due to hypertension, dyslipidaemia, endothelial dysfunction, insulin resistance and coagulopathy. This may increase the risk of atrial fibrillation. However, the Framingham Heart Study did not find a significant risk of atrial fibrillation in hypothyroid subjects. ${ }^{16}$ Overt hypothyroidism may be associated with pericardial effusion and rarely, cardiac tamponade that can present as haemodynamic instability and rhythm disturbances. ${ }^{16}$

\section{Amiodarone-induced thyroid dysfunction}

Amiodarone is a commonly used anti-arhythmic drug that has a high iodine content (37\% of its weight) and can cause thyroid dysfunction in almost $15-20 \%$ of patients. The spectrum of amiodarone-induced thyroid dysfunction can vary from hypothyroidism to thyrotoxicosis. ${ }^{2}$ Amiodarone-induced hypothyroidism (AIH) is due to the persistent inhibition of thyroid function by iodine and is more commonly seen in those with underlying thyroid autoimmunity. ${ }^{17} \mathrm{AlH}$ is more common than thyrotoxicosis. Treatment of persistent $\mathrm{AlH}$ is with levothyroxine replacement. The dose requirement may be high, as amiodarone reduces the peripheral conversion of $\mathrm{T} 4$ to $\mathrm{T3}$.

Amiodarone-induced thyrotoxicosis (AIT) type 1 occurs due to increased thyroid hormone synthesis and secretion (hyperthyroidism), while AIT type 2 is a form of destructive thyrotoxicosis (Table 3). While it is important to differentiate the two types of AIT, it is not always easy to do so. AIT type 1 requires management with anti-thyroid drugs, though dose requirements are usually higher. Potassium perchlorate may be used to increase the response to anti-thyroid drugs, as it inhibits iodine uptake by the thyroid gland. ${ }^{5,17}$ AlT type 2 is treated with glucocorticoids and beta blockers. In mixed or indeterminate forms, combination of anti-thyroid drugs and glucocorticoids should be considered. ${ }^{17}$ Thyroidectomy has been required in rare cases where response to drug therapy is inadequate.

A close discussion between the endocrinologist and the cardiologist is required to decide whether amiodarone can be discontinued or not. While in AlH, amiodarone may be continued along with adequate LT4 treatment, but it would be preferable to discontinue amiodarone in AIT (if possible), especially in patients not responding to anti-thyroid drugs. ${ }^{5,17}$ However, due to the long half-life and fat storage of amiodarone, the effect of the drug may last for a few months, and discontinuation may not have an immediate benefit on thyroid function. Due to the risk of amiodarone-induced thyroid dysfunction, thyroid function should be tested in all patients before starting amiodarone and then after every 6 months for the duration of therapy. ${ }^{5}$

\section{Acromegaly}

Acromegaly is associated with an adverse cardiometabolic profile with hypertension, insulin resistance, diabetes and dyslipidaemia. While the risk of atherosclerotic CVD is somewhat increased in acromegaly, typical structural changes in the heart, including myocardial hypertrophy, inflammation and fibrosis, increase the risk of heart failure as well as arrhythmias. ${ }^{18}$ Acromegalic cardiomyopathy progresses over time from a hyperkinetic stage with increased heart rate and cardiac output to a stage of subclinical diastolic dysfunction, and then eventual systolic dysfunction with reduced ejection fraction and clinical heart failure. ${ }^{18,19}$

An ECG may demonstrate prolonged QT interval, left axis deviation and left ventricular hypertrophy, ST-T wave depression, and septal Q waves. Over $50 \%$ of patients with acromegaly have late potentials that predispose them to rhythm disorders, especially those who are elderly or have cardiomyopathy, valvular heart disease, sleep apnoea or CVD. These include atrial and ventricular ectopic beats, paroxysmal atrial fibrillation or supraventricular tachycardia, sick sinus syndrome, bundle branch block or ventricular tachycardia. ${ }^{5}$ Ventricular arrhythmias are more common in acromegaly, and their incidence increases with age. Complex ventricular ectopy may occur during exercise. ${ }^{19}$ Sustained ventricular tachycardia and sudden cardiac death have been reported in patients with advanced cardiomyopathy, which has become less common due to advances in the management of acromegaly. ${ }^{19}$

The risk of arrhythmias reduces with early detection and effective management of acromegaly. Adequate treatment of the underlying growth hormone-excess state can result in shortening of the QT interval 


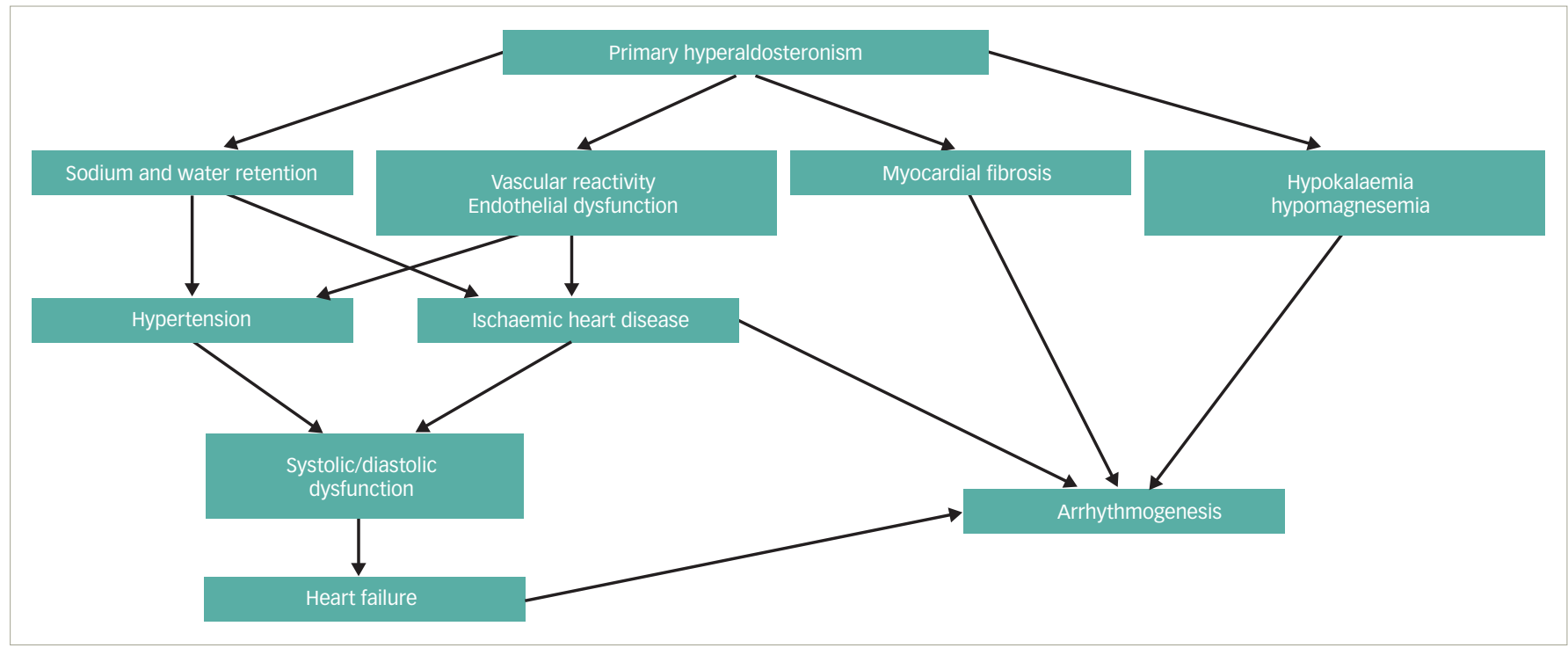

and reduced risk..$^{18}$ Somatostatin analogues have been found to be particularly effective in reducing QT prolongation, and their preoperative use, especially in patients with cardiovascular risk, can improve cardiac dysfunction and increase anaesthesia safety. ${ }^{5}$ Pegvisomant also reduced the prevalence of conduction abnormalities. ${ }^{5}$

\section{Pheochromocytoma}

Catecholamines have positive chronotropic, inotropic and dromotropic effects. Sudden and/or massive release of catecholamines from tumours including pheochromocytoma or paragangliomas can lead to arrhythmias. Cardiovascular risk is significantly increased in pheochromocytoma due to multiple factors: hypertension, endothelial dysfunction, cardiomyopathy, ischaemic heart disease, myocardial stunning and cardiogenic shock. ${ }^{20}$

The typical ECG changes seen in patients with catecholamine-secreting tumours include poor R-wave progression, inverted T waves, QT prolongation, right axis deviation, left axis deviation, left ventricular hypertrophy and left ventricular strain. ${ }^{20}$ Arrhythmias may occur in up to $20 \%$ of patients and can be a presenting feature of pheochromocytoma. ${ }^{5}$ While sinus tachycardia is very common, some patients may develop supraventricular or ventricular tachycardia and sick sinus syndrome. In rare cases, ventricular tachycardia may lead to ventricular fibrillation and sudden death. ${ }^{21}$ Recurrent arrhythmias should raise a suspicion of pheochromocytoma.

While management of pheochromocytoma includes adequate $\alpha$ and $\beta$ blockade followed by surgical removal of the tumour, treatment of arrhythmias can be challenging. A $\beta 1$-selective (cardio-selective) short-acting beta blocker, such as esmolol, is appropriate for the management of atrial fibrillation or flutter, along with concomitant $\alpha$-blockade to prevent hypertensive crisis. ${ }^{21}$

\section{Primary hyperaldosteronism}

Primary hyperaldosteronism increases the risk of arrhythmias via several mechanisms (Figure 3). Bradycardia may result if there is hypoxia or metabolic alkalosis. Hypokalaemia and myocardial fibrosis may result in QT prolongation and increased risk of ventricular tachycardia, polymorphic ventricular tachycardia and ventricular fibrillation. ${ }^{5}$ In addition, there is an increased risk of atrial fibrillation and flutter.
The management of arrhythmias requires prompt correction of underlying electrolyte imbalance (hypokalaemia) and mineralocorticoid antagonists. In cases of aldosterone-producing adenoma, definitive treatment requires surgery.

\section{Cushing's syndrome}

Cardiovascular risk is significantly increased in patients with Cushing's syndrome due to a clustering of risk factors, including central obesity, insulin resistance, diabetes, hypertension, dyslipidaemia and a prothrombotic state. ${ }^{22}$ Cushing's syndrome causes altered sympathetic-parasympathetic balance with reduced heart rate variability, and some patients may have hypokalaemia with metabolic alkalosis. ${ }^{2}$ Literature on arrhythmias in Cushing's syndrome is sparse; however, cases of atrial fibrillation in Cushing's syndrome have been reported. ${ }^{23-25}$ Carney's complex, comprising of café-au-lait spots, lentigines, atrial myxomas, breast myxomas or ductal adenomas, primary pigmented nodular adrenal hyperplasia, and causing Cushing's syndrome and other endocrine tumours, may be associated with atrial arrhythmias. ${ }^{22}$

\section{Adrenal insufficiency}

Glucocorticoids are needed for the maintenance of membrane calcium transport in the cardiac sarcoplasmic reticulum. ECG may reveal sinus bradycardia, low voltage QRS complex, prolonged PR or QT intervals, and T-wave inversion. ${ }^{5}$ Patients with adrenal insufficiency may have hyponatraemia, hyperkalaemia and hypoglycaemia that increases the risk of rhythm disturbances such as ventricular tachycardia or ventricular fibrillation, though they have not been commonly reported.

\section{Hypercalcaemia}

Hypercalcemia may result from parathyroid hormone-dependent or -independent causes, and is associated with a reduction in the plateau phase of the ventricular action potential and effective refractory period, especially when serum calcium is $>12 \mathrm{mg} / \mathrm{dL} .{ }^{26}$ Typical ECG changes that are seen in hypercalcaemia include shortened QT interval, high amplitude QRS complex and widened or biphasic T wave. Hypercalcaemia increases the risk of arrhythmias ranging from sinus arrest, supraventricular tachycardia, atrial fibrillation, ventricular bigeminy, ventricular tachycardia and ventricular fibrillation. ${ }^{5}$ Very high serum calcium can cause cardiac arrest. ${ }^{27}$ Prompt correction of hypercalcaemia with saline diuresis, loop diuretics, bisphosphonates and/or dialysis is important to reduce the 
risk of life-threatening arrhythmias. In addition, there is increased risk of hypertension, impaired glucose tolerance and CVD in patients with primary hyperparathyroidism, which increases the risk of left ventricular hypertrophy and structural heart disease.

\section{Hypocalcaemia}

The hallmark ECG abnormality in patients with hypocalcaemia is QT prolongation due to the prolongation of the plateau phase of ventricular action potential. ${ }^{28}$ However, in practice, torsades de pointes or life-threatening tachyarrhythmias are infrequent but reported. ${ }^{29}$ Severe hypocalcaemia may be associated with T-wave changes, such as flattened T wave, terminal inversion or deeply inverted T waves. ${ }^{28}$ Rarely, ST segment elevation may be seen due to coronary artery spasm.

Hypocalcaemia may rarely be associated with dilated cardiomyopathy and heart failure due to impaired excitation-contraction coupling. correction of serum calcium levels using intravenous calcium infusion can reverse the ECG changes and improve myocardial contractility. There is often a progressive decrease in QTc interval, with a rise in serum calcium levels as hypocalcaemia is corrected.

\section{Sex steroids and cardiac conduction}

Sex hormones, including testosterone, progesterone and oestradiol, can modulate ventricular repolarization. Testosterone and progesterone shorten the action potential and QT interval; on the other hand, oestradiol prolongs it. 5,30 Women tend to have a higher resting heart rate and shorter PR and QRS intervals, but a longer QT interval. ${ }^{30}$ The menstrual phase also impacts the duration of QT interval, with a longer QT interval in the follicular phase than the luteal phase (where progesterone secretion opposes the QT prolonging effect of oestrogen). ${ }^{31}$ Longer repolarization makes women more susceptible to torsades de pointes. ${ }^{30}$ On the other hand, men are more susceptible to Brugada syndrome and atrial fibrillation..$^{30}$ While combination oral contraceptives or long-acting contraception is not associated with arrhythmia risk, QT prolongation has been reported with drospirenone. ${ }^{30}$ Selective oestrogen-receptor modulators that are used in breast cancer treatment may also cause QT prolongation and increase the risk of torsades de pointes. ${ }^{32}$

Men with hypogonadism are at increased risk of QT prolongation. In addition, androgen deprivation therapy and long-acting gonadotropin-releasing hormone analogue therapy used in men with prostate cancer can also cause QT prolongation. ${ }^{32}$ Anabolic steroid abuse in athletes and body builders is also associated with increased risk. ${ }^{31}$ Table 4 shows various endocrine drugs that can cause QT
Table 4: Endocrine therapies associated with QT prolongation

\begin{tabular}{|l|l|}
\hline Diabetes therapy & $\begin{array}{l}\text { Agents causing QT prolongation } \\
\text { Teneligliptin } \\
\text { Hydroxychloroquine } \\
\text { Remogliflozin }\end{array}$ \\
\hline Acromegaly & Octreotide \\
\hline Progesterone & Drospirenone \\
\hline Anabolic steroid & Anabolic androgen abuse in body builders and athletes \\
\hline $\begin{array}{l}\text { Cancer } \\
\text { chemotherapy }\end{array}$ & $\begin{array}{l}\text { Selective oestrogen-receptor modulator in breast cancer } \\
\text { (toremifene }>\text { tamoxifen) } \\
\text { Androgen deprivation therapy in prostate cancer } \\
\text { GnRH analogue in prostate cancer }\end{array}$ \\
\hline
\end{tabular}

GnRH = gonadotrophin-releasing hormone.

prolongation. Concomitant factors that increase the risk of ventricular arrhythmia and sudden death in patients with prolonged QT include hypokalaemia, pre-existent cardiac disease, cardiac autonomic neuropathy, underlying QT prolongation and hypoglycaemia.

\section{Conclusion}

Cardiac rhythm is closely regulated by the neuroendocrine axis, with an intricate balance between the sympathetic and parasympathetic nervous systems. In addition, various cardiac reflexes and hormones mediate changes in heart rate, cardiac conduction and contractility to meet the oxygen and nutritional demands of the body. Endocrine and metabolic disorders can cause rhythm disorders, both as a direct result of hormone excess or deficiency states or indirectly due to structural or ischaemic heart disease and electrolyte or mineral imbalance.

There is a clear need for endocrine and metabolic vigilance in patients presenting with rhythm or conduction abnormalities, as the identification and appropriate management of underlying endocrinopathy can significantly reduce the risk. At the same time, cardiac vigilance is required during the management of endocrine disorders such as diabetes, acromegaly, pheochromocytoma, Cushing's syndrome, primary hyperaldosteronism and hyperparathyroidism. While these disorders are associated with an increased risk of ischaemic heart disease, heart failure and cardiomyopathy, the risk of arrhythmias is also increased. In addition, endocrine therapies may also impact the arrhythmic risk. Therefore, there is a need for close collaboration between the endocrinologist and the cardiologist. $\square$
1. Purtell K, Roepke TK, Abbott GW. Cardiac arrhythmia and thyroid dysfunction: a novel genetic link. Int I Biochem Cell Biol. 2010;42:1767-70.

2. Rhee SS, Pearce EN. The endocrine system and the heart: A review. Rev Esp Cardiol. 2011;64:220-31.

3. Gordan R, Gwathmey JK, Xie LH. Autonomic and endocrine control of cardiovascular function. World $\mathrm{I}$ Cardiol. 2015:7:204-14.

4. Shen MJ, Zipes DP. Role of the autonomic nervous system in modulating cardiac arrhythmias. Circ Res. 2014:114:1004-21 modulating cardiac ar oyth as. Circ Res. 2014,114.1004-21.

- Gorek B, Borlani G, Dan GA, et al. European Heart Rhythm Association (EHRA) position paper on arrhythmia managemen and device therapies in endocrine disorders, endorsed by Asia Pacific Heart Rhythm Society (APHRS) and Latin American Heart Rhythm Society (LAHRS). Europace. 2018;20:895-6.

6. Kalra S, Kishor K, Batra A, Aggarwal S. Atrial fibillation in diabetes: Need for cardiovigilance. J Pak Med Assoc. 2019;69:437-9.

Echouffo-Tcheugui JB, Shrader P, Thomas L, et al. Care patterns and outcomes in atrial fibrillation patients with and without diabetes: ORBIT-AF Registry. J Am Coll Cardiol. 2017;70:1325-35.

8. Grisanti LA. Diabetes and arrhythmias: pathophysiology, mechanisms and therapeutic options. Front Physiol. 2018:9:1669.
9. Erande S, Sarwardekar S, Desai B. QT/QTC safety and efficacy evaluation of teneligliptin in Indian type 2 diabetes mellitus patients: the "thorough QT/QTC study" (Q-SET study). Diabetes Metab Syndr Obes. 2019;12:961-7.

10. Chen CY, Wang FL, Lin CC. Chronic hydroxychloroquine use associated with QT prolongation and refractory ventricular arrhythmia. Clin Toxicol (Phila). 2006;44:173-5.

11. Lorenz M, Lawson F, Owens D, et al. Differential effects of glucagon-like peptide-1 receptor agonists on heart rate. glucagon-like peptide-1 receptor

12. Monami M, Nreu B, Scatena A, et al. Glucagon-like peptide-1 receptor agonists and atrial fibrillation: a systematic review and meta-analysis of randomised controlled trials. J Endocrino Invest. 2017:40:1251-8.

13. Sivanandy $P$, Chunmey L. Management of thyrotoxicosis with atrial fibrillation- a case report. J Young Pharm. 2017;9:616-9.

4. Kalra S, Kishor K, Khandelwal D. Atrial fibrillation: The endocrine connection. J Pak Med Assoc. 2019;69:1746-9.

5. Udovcic M, Pena RH, Patham B, et al. Hypothyroidism and the heart. Methodist Debakey Cardiovasc J. 2017;13:55-9.

16. Kim EJ, Lyass A, Wang N, et al. Relation of hypothyroidism and incident atrial fibrillation (from the Framingham Heart Study). Am Heart J. 2014:167:123-6.

17. Bogazzi F, Bartalena L, Martino E. Approach to the patient with amiodarone-induced thyrotoxicosis. I Clin Endocrinol Metab. 2010;95:2529-35

18. Ramos-Levi AM, Marazuela M. Bringing cardiovascular comorbidities in acromegaly to an update. How should we diagnose and manage them? Front Endocrinol (Lausanne). 2019;10:120.

19. Mizera L, Elbaum M, Daroszewski J, Bolanowski M. Cardiovascular complications of acromegaly. Acta Endocrinol (Buchar). 2018:14:365-74.

20. Galetta F, Franzoni F, Bernini G, et al. Cardiovascular complications in patients with pheochromocytoma: mini-review. Biomed Pharmacother. 2010;64:505-9.

21. Zhang MM, Mao W, WU D, Liu P. Pheochromocytoma with ventricular tachycardia as the presenting symptom. Chin Med J (Engl). 2016;129:1505-6.

22. De Leo M, Pivonello $\mathrm{R}$, Auriemma RS, et al. Cardiovascular disease in Cushing's syndrome: heart versus vasculature. Neuroendocrinology. 2010;92(Suppl. 1):50-4.

23. Singer $\mathrm{E}$, Strohm $\mathrm{S}$, Gobel U, et al. Cushing's disease, hypertension, and other sequels. Hypertension. 2008;52:1001-5

24. Shand J. An unusual cause of atrial fibrillation. The New Zealand Medical Student Journal. 2014:18-9:12-3.

25. Koracevic $\mathrm{G}$, Mićić $\mathrm{S}$, Stojanović $\mathrm{M}$, et al. High likelihood for atrial 


\section{Review Arrhythmia}

fibrillation in Cushing's syndrome. Eur Rev Med Pharmacol Sci. 2020;24:1391-7

26. Vella A, Gerber TC, Hayes DL, et al. Digoxin, hypercalcaemia,

and cardiac conduction. Postgrad Med J. 1999;75:554-6.

27. Pepe J, Curione M, Morelli S, et al. Arrhythmias in primary

hyperparathyroidism evaluated by exercise test. Eur $J$

Clin Invest. 2013:43:208-14.
28. Cecchi E, Grossi F, Rossi M, et al. Severe hypocalcemia and life-threatening ventricular arrhythmias: case report and proposal of a diagnostic and therapeutic algorithm. Clin Cases Miner Bone Metab. 2015;12:265-8.

29. Chhabra ST, Mehta S, Chhabra S, et al. Hypocalcemia presenting as life threatening torsades de pointes with prolongation of QTC interval. Indian J Clin Biochem. 2018;33:235-8.
30. Ravens U. Sex differences in cardiac electrophysiology. Can J Physiol Pharmacol. 2018:96:985-90.

31. Gonzalez $C D$, de Sereday M, Sinay I, Santoro S. Endocrine therapies and QTC prolongation. Curr Drug Saf. 2010;5:79-84.

32. Barber M, Nguyen LS, Wassermann J, et al. Cardiac arrhythmia considerations of hormone cancer therapies. Cardiovasc Res. 2019;115:878-94. 